\title{
Case Report: A Laparoscopic Treated Case of Deep Infiltrating Endometriosis with Vaginal Invasion Diagnosed by Vaginal Cytology
}

\author{
Kenji Niwa1 ${ }^{*}$, Ryuichiro Yano 2 , Yui Hatanaka3 ${ }^{3}$, Sakae Mori' ${ }^{3}$, Yoshio Yamaguchi3, \\ Minako Mori' ${ }^{2}$, Yoko Ueda ${ }^{1}$, Takuji Tanaka ${ }^{4}$ \\ ${ }^{1}$ Department of Obstetrics \& Gynecology, Gujo City Hospital, Gujo City, Gifu, Japan \\ ${ }^{2}$ Department of Obstetrics \& Gynecology, Gifu University Graduate School of Medicine, Gifu City, Gifu, Japan \\ ${ }^{3}$ Section of Laboratory Medicine, Gujo City Hospital, Gujo City, Gifu, Japan \\ ${ }^{4}$ Department of Diagnostic Pathology (DDP) \& Research Center of Diagnostic Pathology (RC-DiP), \\ Gifu Municipal Hospital, Gifu City, Gifu, Japan \\ Email: ${ }^{*}$ kniwa.gujo913@gmail.com
}

Received 28 May 2015; accepted 6 July 2015; published 9 July 2015

Copyright (C 2015 by authors and Scientific Research Publishing Inc.

This work is licensed under the Creative Commons Attribution International License (CC BY). http://creativecommons.org/licenses/by/4.0/

(c) (i) Open Access

\begin{abstract}
A recently observed case of deep infiltrating endometriosis with its vaginal invasion diagnosed by vaginal cytology is reported. A 28-year-old Japanese woman complained of a severe dysmenorrhea. Left ovarian endometriotic cyst and deep infiltrating endometriosis were suspected. Two redspots were also present in the posterior fornix of vagina. Vaginal cytology with cytokeratin (CK) 7-immunocytochemistry suggested the presence of infiltrating endometriosis. She underwent left ovarian cystectomy and resection of thickened uterosacral ligaments with vaginal endometriotic spots, under a laparoscope, based on the diagnosis of endometriotic cyst. Histopathology revealed DIE with vaginal invasion. Immunostaining for CK7 was positive for endometriotic cyst, while CK20 was negative. The stromal cells in endometriosis were immunohistochemically positive for CD10. The vaginal fornix was well-healed one month later the surgery. Postoperatively, she got pregnant with her natural intercourse and delivered a healthy $2138 \mathrm{~g}$ girl at 36 weeks of gestation.
\end{abstract}

\section{Keywords}

Vaginal Endometriosis, Cytology, Immunohistochemistry, Laparoscopic Surgery

\footnotetext{
${ }^{*}$ Corresponding author.

How to cite this paper: Niwa, K., Yano, R., Hatanaka, Y., Mori, S., Yamaguchi, Y., Mori, M., Ueda, Y., and Tanaka, T. (2015) Case Report: A Laparoscopic Treated Case of Deep Infiltrating Endometriosis with Vaginal Invasion Diagnosed by Vaginal Cytology. Open Journal of Pathology, 5, 84-89. http://dx.doi.org/10.4236/ojpathology.2015.53012
} 


\section{Introduction}

Endometriosis is defined as the presence of endometrial glands and stroma abnormal located outside the uterine cavity. It is a benign gynecological disorder affecting $6 \%-10 \%$ of all women of reproductive age and presents an important cause of infertility [1]. Deep infiltrating endometriosis (DIE) is a particular form of endometriosis that extends $>5 \mathrm{~mm}$ under the peritoneal surface [2]. The lesions develop in the form of retroperitoneal nodules that consist histologically of endometrial epithelium and stroma [3]. DIE nodules represent a real operative challenge due to common involvement of vital structures, such as ureter, bowel, vessels and nerves. Laparoscopy appears to be an ideal tool to perform such surgery, offering the advantages of magnification, accurate hemostasis, precise dissection and fertility [4].

Lower genital tract endometriosis, such as vagina and uterine cervix, might be more common than is generally realized. The theory of transplantation is favored for extra-pelvic endometriosis, but the disease could also arise through a direct implantation or metaplasia of urachus remnants [5]. Although the definitive diagnosis is made by biopsy of fine needle aspiration cytology showing the usual morphological features of endometriosis [6] [7], the exfoliative cytology with CK7 from the vaginal nodes was useful to differential diagnose from neoplastic lesions [8] [9].

Here our recently observed case of ovarian endometriotic cyst and DIE with vaginal endometriosis that were diagnosed by vaginal cytology with immunocytochemistry of CK7 is reported. After the surgery, she got pregnant and was delivered a healthy girl after surgical treatment.

\section{Clinical Case}

A 27-year-old Japanese woman, gravida 0, visited to our hospital complaining of severe dysmenorrhea. Her medical history was unremarkable. She did not take any oral contraceptives and had regular menstrual cycles. Vaginal examination revealed two reddish nodules of approximate $1 \mathrm{~mm}$ in diameter located at the posterior vaginal fornix (Figure 1). A left ovarian cyst and recto-vaginal nodules were also detected, suggesting a DIE. Imprint cytology for two nodules at the posterior vaginal fornix suggested the endometriotic cells (Figure 2(A)). To determine the presence of endometriotic cells, immunocytochemistry of cytokeratine (CK) 7, was performed using an antibody against CK7 (DAKO, Denmark). The endometriotic cells expressed cytoplasmic positivity for CK7 (Figure 2(B)). Left ovarian endometriotic cyst was suggested on T2-weighted MRI (Figure 3). The thickened uterosacral ligaments were suspected on other MRI. She was diagnosed lt-ovarian endometriotic cyst and a DIE with thickened uterosacroal ligaments and vaginal endometriosis, but she did not wish surgical treatment at that time.

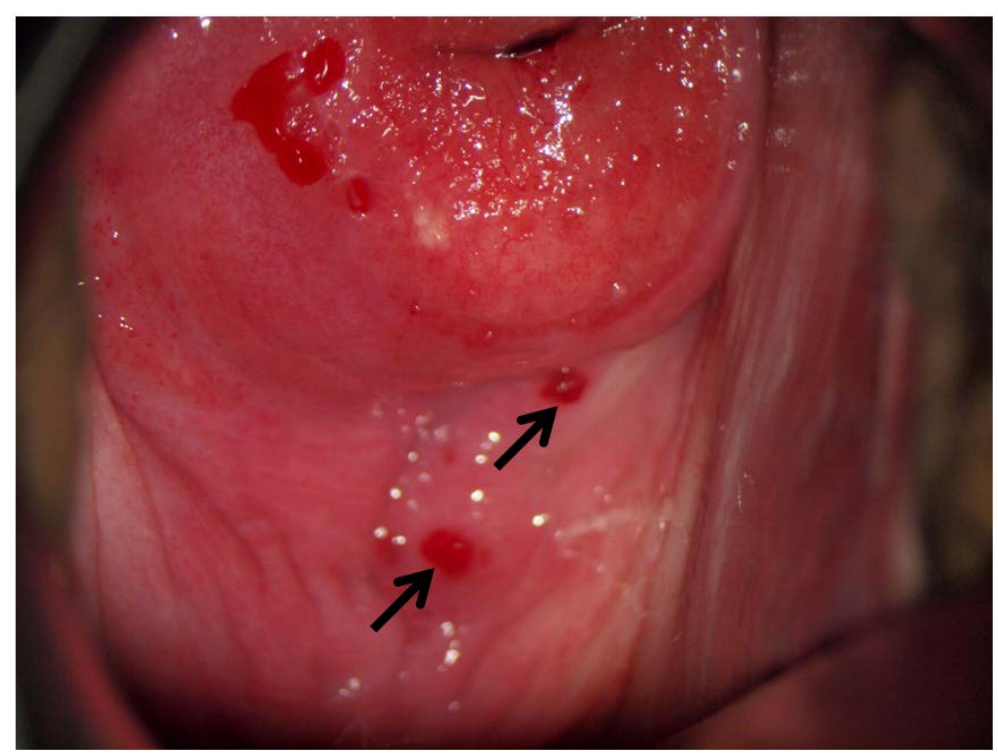

Figure 1. Colposcopic examination of the posterior fornix shows two reddish nodules (Approximate $1 \mathrm{~mm}$ in diameter), suggesting vaginal endometriosis. 


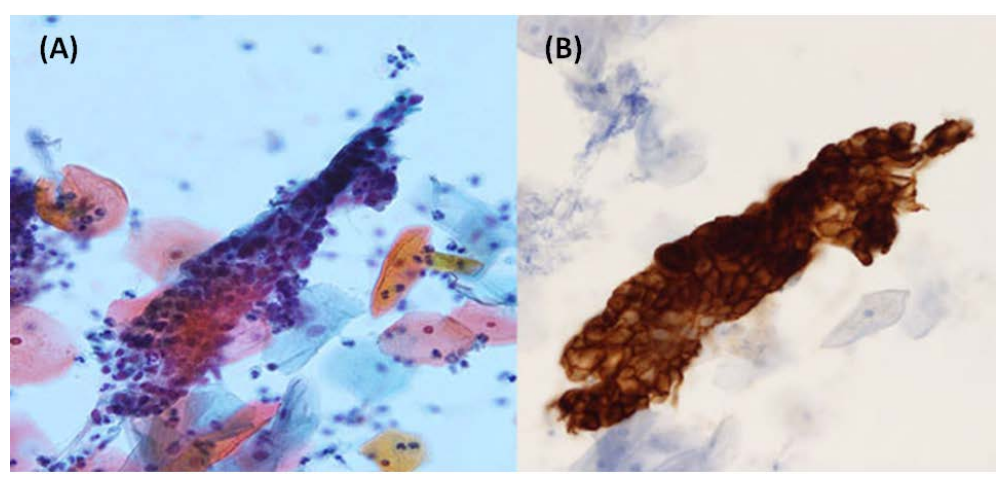

Figure 2. (A) On imprint cytology from the nodes at the posterior vaginal fornix, the endometriosis-like cells are present (Papanicolaou stain, original magnification: $\times 100$ ); (B) CK7 immunocytochemistry shows positive reaction in the cytoplasm of endometriosis-like cells (CK7 immunocytochemistry, original magnification: $\times 100$ ).

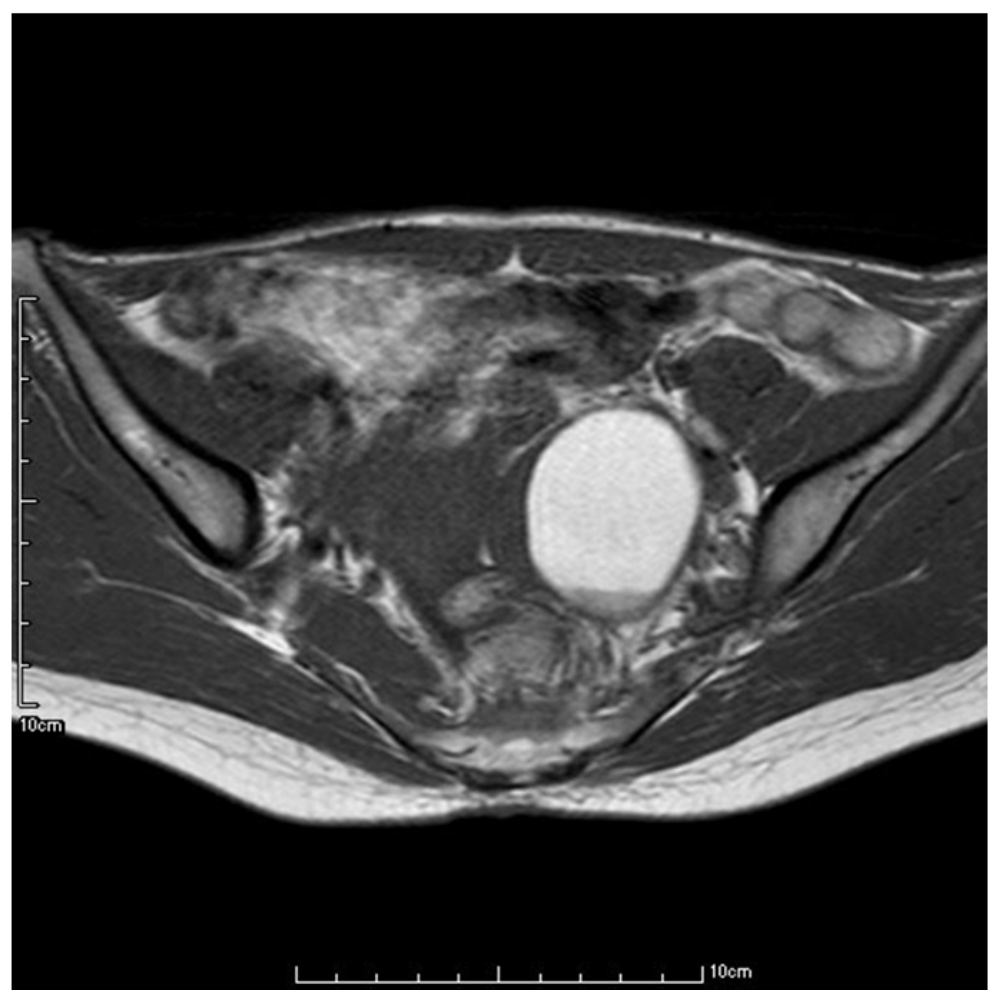

Figure 3. Left-ovarian endometriotic cyst is detected on the T2-weighted MRI, suggesting endometriotic cyst.

Five months after her first visit, she had noticed a reinforcement of dyspareunia and non-menstrual pain. The size of the left ovarian endometriotic cyst has enlarged in transvaginal ultrasonography. She wished a laparoscopic surgery to remove the left ovarian endometriotic cyst. Under the working diagnosis of the lt-endometriotic cyst and DIE with vaginal endometriosis, she underwent the left ovarian cystectomy and the thickened uterosacral ligaments with vaginal endometriotic spots, under a laparoscope (Figure 4(A)). Vaginal wall was sutured continuously under a laparoscope and the resected tissues (Figure 4(B) and Figure 4(C)) were fixed in 10\% buffered formalin (Nacalai Tesque Co. ltd., Kyoto, Japan) for 3 days and embedded in paraffin (Wako Pure Chemical, Osaka, Japan). Four $\mu$ m-thick sections were made and stained with hematoxylin and eosin (Muto pure chemical Co. ltd., Tokyo, Japan) for histopathological examination. On microscopic examination, glands with stromal cells were found in the all resected tissues (Figure 5). To determine the presence of endometriotic cells, immunohistochemistry was performed using antibodies against CK7 (DAKO, Denmark), CK20 (DAKO, Denmark), and CD10 (Novacastra, Leica Biosystem, Newcastle, UK). CD10 was weakly positive in the stromal 


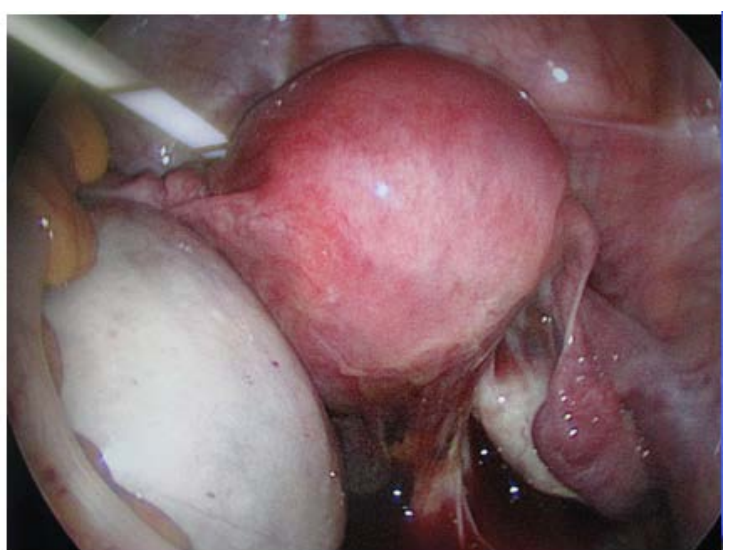

(A)

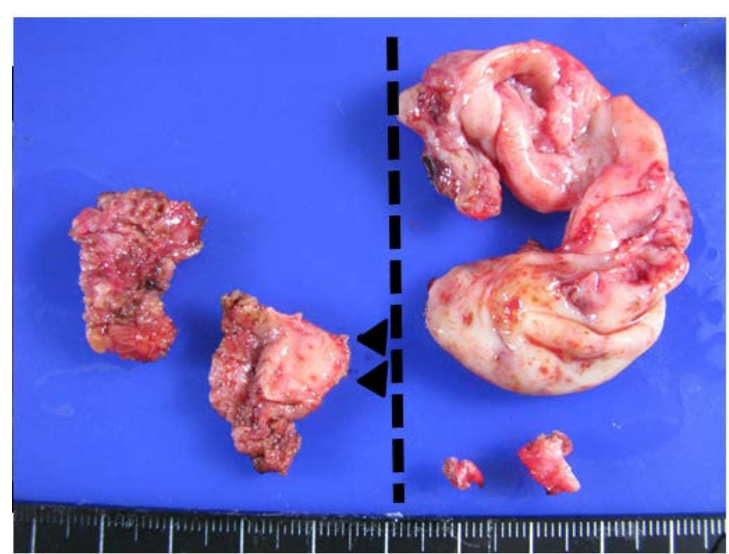

(B)

(C)

Figure 4. (A) Leftt-ovarian cyst, possibly the endometriotic cyst, and thickened uterosacral ligaments are seen under a laparoscope; Macroscopic view of the resected (B) uterosacral ligamnets with vaginal endometriosis and (C) left-ovarian cyst. Vaginal reddish spots were present (arrow heads).

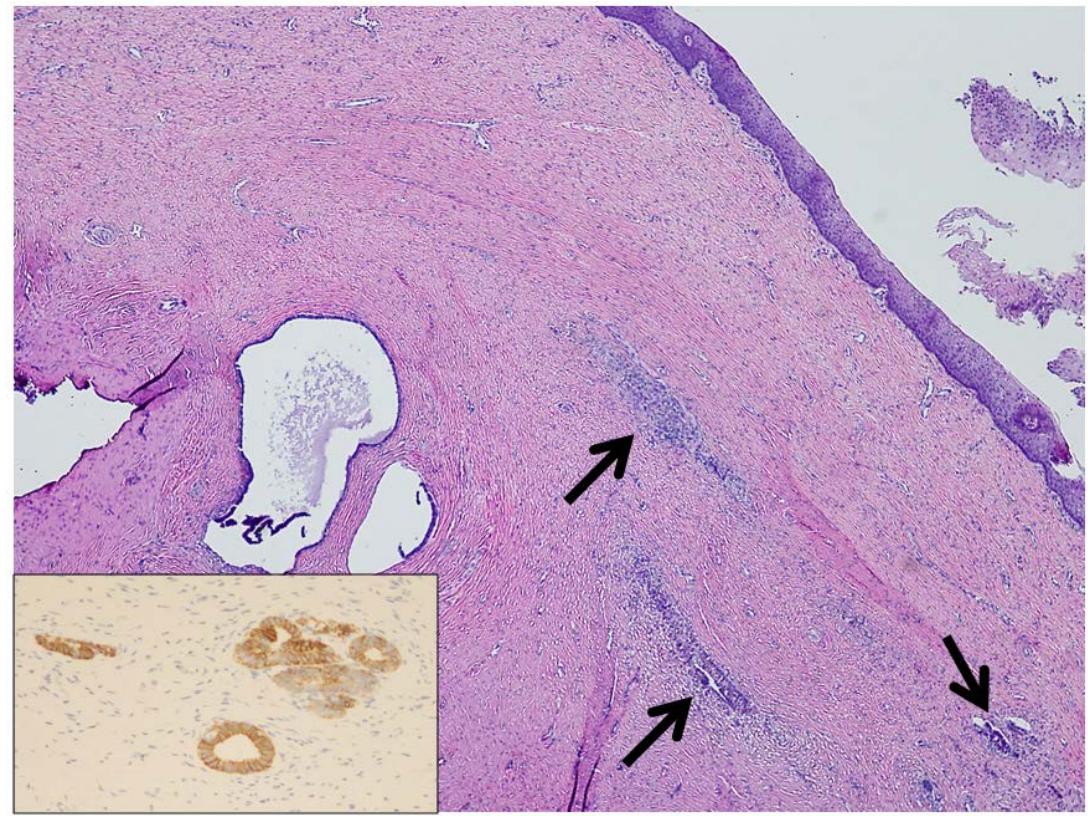

Figure 5. Histopathology of the resected specimens shows endometrioses. In the vagina endometriotic glands (arrows) with the stroma were present under the subcutaneous (original magnification: $\times 40$ ). Insert: CK7 immunohistochemistry shows that positive reaction is present in the cytoplasm of endometriotic glands (original magnification: $\times 100$ ).

component, and CK7 (Figure 5 insert) was positive in the epithelial components, respectively. CK20 was negative in the stromal and epithelial components. Thus, our pathological diagnosis was made as lt-ovarian endometriotic cyst and DIE including uterosacral ligaments and vaginal endometrioses.

Postoperative recovery was uneventful, and she was discharged on the $3^{\text {rd }}$ day after the laparoscopic surgery. One month after the surgery, the vaginal fornix was well-healed (Figure 6). Pregnancy was permitted for her three months after the surgery, and she got pregnant with her natural intercourse six months after the surgery. The gestational course was uneventful, but the fetal growth was suspected a little low range (within $10 \%$ range) under an ultrasonic evaluation. At 36 weeks of gestation, she showed pre-term rupture of membrane, and showed frequent variable decelerations probably due to cord compression. She underwent an emergent cesarean section, and was delivered a healthy $2138 \mathrm{~g}$ girl. She had no complaints of dysmenorrhea and dyspareunia two years after the surgery. 


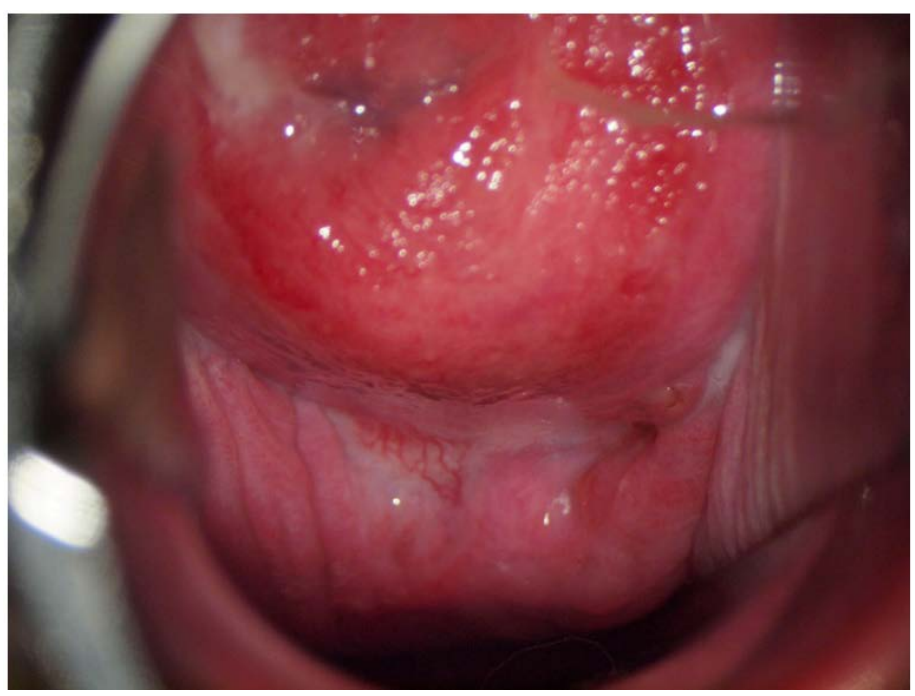

Figure 6. The colposcopy reveals that the vaginal wall in the posterior fornix was healed sufficiently one month after the surgery.

\section{Discussion}

A laparoscopic treated case of ovarian endometriotic cyst and DIE with vaginal endometriosis was reported. The vaginal cytology showing positive reactivity of CK7 in the glandular cells was useful for the diagnosis of the vaginal endometriosis.

Uterine cervical and vaginal endometrioses were reported to be $3 \%$ of all cases of endometriosis [10]. Although superficial cervical and vaginal endometrioses were thought to be strong predilection for the sites of obstetrical and operative trauma [11], the vaginal endometriosis in this case was suggested to be direct invasion from the DIE with thickened uterosacral ligaments by the histopathological examinations. Pelvic endometrioses show positive reaction for CK7 in the endometriotic cells and that for CD10 in the stromal cells. On the other hand, the endometriotic cells are negative for CK20 [8] [9]. In our case, immunostainings for CK7/20 and CD10 in the cytological and histopathogical specimens were useful for the diagnosis of ectopic endometriosis, especially in the vagina.

DIE is a particular form of endometriosis, extending over $5 \mathrm{~mm}$ under the peritoneal surface [1]. As DIE nodules are generally rich in nerve fibers [12], patients with DIE suffered from severe pelvic pain, such as dysmenorrhea and deep dyspareunia [13]-[15]. The medical therapies may temporally alleviate painful symptoms, however recurrence rates after the discontinuation are reported to be high [4] [16]. Thus, radical surgical excisions for DIE lesion are thought to be the mainstay of the treatment for this type of endometriosis. Although laparoscopic approaches appear to be the ideal tool to perform such surgeries for DIE, approaches for DIE nodules are real operative challenge due to common involvement of retroperitoneal structures (ureter, bowel, vessels and nerves) [4]. After the surgery, half of the patients wishing for her babies were reported to conceive spontaneously, suggesting a relationship between DIE and infertility [17]. In the present case, she got pregnant with her natural intercourse six months after the surgery, and was delivered a healthy girl.

\section{Conclusion}

Our recently observed case of ovarian endometriotic cyst and DIE with vaginal endometriosis is presented. Vaginal cytology with CK7-immunocytochemistry was useful for diagnosis. After left ovarian cystectomy and laparoscopic resection of thickened uterosacral ligaments with vaginal endometriotic spots, histopathological examination with CK7-, CK20- and CD10-immunohistochemistry confirmed cytological diagnosis. After surgery, she successfully delivered a healthy girl.

\section{Competing Interests}

The authors declare that they have no competing interests. 


\section{Acknowledgements}

We thank the editor and reviewers for their constructive comments, which helped us to improve the manuscript. This case report was approved by the patient verbal consent.

\section{References}

[1] Giudice, L.C. and Kao, L.C. (2004) Endometriosis. The Lancet, 364, 1789-1799. http://dx.doi.org/10.1016/S0140-6736(04)17403-5

[2] Koninckx, P.R., Meuleman, C., Demeyere, S., Lesaffre, E. and Cornillie, F.J. (1991) Suggestive Evidence That Pelvic Endometriosis Is a Progressive Disease, Whereas Deeply Infiltrating Endometriosis Is Associated with Pelvic Pain. Fertility and Sterility, 55, 759-765.

[3] Anaf, V., Simon, P., El Nakadi, I., Fayt, I., Buxant, F., Simonart, T., Peny, M.O. and Noel, JC. (2000) Relationship between Endometriotic Foci and Nerves in Rectovaginal Endometriotic Nodules. Human Reproduction, 15, 1744-1750. http://dx.doi.org/10.1093/humrep/15.8.1744

[4] Protopapas, A., Giannoulis, G., Chatzipapas, I., Athanasiou, S., Grigoriadis, T., Haidopoulos, D., Loutradis, D. and Antsaklis, A. (2014) Posterior Deep Infiltrating Endometriotic Nodules: Operative Considerations According to Lesion Size, Location, and Geometry, during One’s Learning Curve. ISRN Obstetrics and Gynecology, 20, 1-8. http://dx.doi.org/10.1155/2014/853902

[5] Witz, C.A. (1999) Current Concepts in the Pathogenesis of Endometriosis. Clinical Obstetrics and Gynecology, 42, 566-585. http://dx.doi.org/10.1097/00003081-199909000-00013

[6] Mederios, F.C., Cavalcante, D.I.M., Mederios, M.A.S. and Eleutério Jr., J. (2010) Fine-Needle Aspiration Cytology of Scar Endometriosis: Study of Seven Cases and Literature Review. Diagnostic Cytopathology, 39, 18-21. http://dx.doi.org/10.1002/dc.21319

[7] Oliveira-Filho, M., Rao, V.S., Eleutério Jr., J. and Medeiros, F.C. (2013) Fine Needle Aspiration Cytology: A Tool to Diagnose Cervical and Vaginal Endometriosis in Low-Income Places. Acta Cytologica, 57, 203-206. http://dx.doi.org/10.1159/000345898

[8] Al-Khawaja, M., Tan, P.H., MacLennan, G.T., Lopez-Beltran, A., Montironi, R. and Cheng, L. (2008) Ureteral Endometriosis: Clinicopathological and Immunohistochemical Study of 7 Cases. Human Pathology, 39, 954-959. http://dx.doi.org/10.1016/j.humpath.2007.11.011

[9] Dharan, M. (2009) The Adjunctive Value of CD10 Immunostaining on Cell Block Preparations in Pelvic Endometriosis. Acta Cytologica, 53, 625-629. http://dx.doi.org/10.1159/000325402

[10] Clement, P.B., Young, R.H. and Scully, R.E. (1990) Stromal Endometriosis of the Uterine Cervix. A Variant of Endometriosis that May Simulate a Sarcoma. The American Journal of Surgical Pathology, 14, 449-455. http://dx.doi.org/10.1097/00000478-199005000-00004

[11] Biscotti, C.V. and Ray, N. (2010) Papanicolaou Tests Associated with Cervical Mucosal Endometriosis: An Analysis of Cellular Features and Comparison to Endocervical Adenocarcinoma in Situ. Diagnostic Cytopathology, 38, 551-554.

[12] Wang, G., Tokushige, N., Markham, R. and Fraser, I.S. (2009) Rich Innervation of Deep Infiltrating Endometriosis. Human Reproduction, 24, 827-834. http://dx.doi.org/10.1093/humrep/den464

[13] Chapron, C., Fauconnier, A., Dubuisson, J.B., Barakat, H., Vieira, M. and Bréart, G. (2003) Deep Infiltrating Endometriosis: Relation between Severity of Dysmenorrhoea and Extent of Disease. Human Reproduction, 24, 760-766. http://dx.doi.org/10.1093/humrep/deg152

[14] Chapron, C., Barakat, H., Fritel, X., Dubuisson, J.B., Bréart, G. and Fauconnier, A. (2005) Presurgical Diagnosis of Posterior Deep Infiltrating Endometriosis Based on a Standardized Questionnaire. Human Reproduction, 20, 507-513. http://dx.doi.org/10.1093/humrep/deh627

[15] Ferrero, S., Abbamonte, L.H., Giordano, M., Ragni, N. and Remorgida, V. (2007) Deep Dyspareunia and Sex Life after Laparoscopic Excision of Endometriosis. Human Reproduction, 22, 1142-1148. http://dx.doi.org/10.1093/humrep/del465

[16] Vercellini, P., Pietropaolo, G., De Giorgi, O., Pasin, R., Chiodini, A. and Crosignani, P.G. (2005) Treatment of Symptomatic Rectovaginal Endometriosis with an Estrogen-Progestogen Combination versus Low-Dose Norethindrone Acetate. Fertility and Sterility, 84, 1375-1387. http://dx.doi.org/10.1016/j.fertnstert.2005.03.083

[17] Donnez, J. and Squifflet, J. (2010) Complications, Pregnancy and Recurrence in a Prospective Series of 500 Patients Operated on by the Shaving Technique for Deep Rectovaginal Endometriotic Nodules. Human Reproduction, 25, 1949-1958. http://dx.doi.org/10.1093/humrep/deq135 